\title{
Escoliose idiopática do adolescente: análise do grau de correção obtido com 0 uso de parafusos pediculares
}

\author{
Adolescent idiopathic scoliosis: analysis of the degree of correction obtained with \\ pedicle screws
}

Luciano Miller Reis Rodrigues', Adriano Masayuki Yonezaki', Fabrício Hidetoshi Ueno', Rodrigo Junqueira Nicolau', Luiz Carlos de Abreu', Edgar Valesin Santiago Filho', André E Marcondes César ${ }^{1}$

\section{Resumo}

Objetivo: Avaliar o grau de correção obtido durante o tratamento cirúrgico da escoliose idiopática do adolescente, por meio da estabilização com parafusos pediculares. Método: Entre 2006 e 2008, foram avaliados 21 pacientes com escoliose idiopática do adolescente, os quais foram submetidos ao tratamento cirúrgico, com seguimento médio de 2 anos. Este estudo foi feito por meio de radiografias no plano frontal com mensuração da correção da deformidade pelo método de Cobb. Foi analisada, também, a rotação da vértebra apical pós-cirúrgica através da classificação de Nash e Moe. Resultados: Foi identificada melhora média de 24,1 graus nas deformidades pós-operatórias torácicas e de 18,78 graus nas deformidades lombares. Isso reflete em uma melhora do ângulo de Cobb de 61,36\% para as curvas torácicas e de 53,65\% para as curvas lombares. Também foi constatada melhora de 1 grau na Classificação de Nash e Moe presente em 11 (52,38\%) pacientes do estudo. Nos demais 10 (47,62\%) pacientes, não houve alteração do grau de rotação pré e pós-operatório na Classificação de Nash e Moe. Conclusões: A fixação com parafusos pediculares, tanto na região lombar quanto torácica, mostrou-se um método seguro com um bom grau de correção nos três planos (sagital, coronal e rotacional).

Palavras-chave: Escoliose/cirurgia; adolescente; doenças da coluna vertebral.

\section{Abstract}

Objective: To assess the degree of correction obtained from surgical stabilization of adolescent idiopathic scoliosis with pedicle screws. Method: Between 2006 and 2008, 21 adolescents with idiopathic scoliosis submitted to surgical treatment were assessed, with a mean follow-up of two years. The assessment was based on frontal plane X-rays with measurement of the deformity correction by the Cobb method. The post-surgical apical vertebra rotation was also assessed, using the Nash-Moe classification. Results: Fast postoperative improvement of 24.1 degrees for thoracic and 18.78 degrees for lumbar deformities was identified. This reflects an improvement of the Cobb angle of $61.36 \%$ for thoracic curves and $53.65 \%$ for lumbar curves. One-degree improvement of the Nash-Moe classification in 11 (52.38\%) study patients was also verified. The remaining 10 (47.62\%) patients showed no change in the pre and post-surgical rotation degree, in the Nash-Moe classification. Conclusion: The fixation with pedicle screws, either lumbar or thoracic, has been shown to be a safe method, resulting in a good degree of correction on the three planes (sagittal, coronal and rotational).

Keywords: Scoliosis/surgery; adolescent; spinal diseases.

Recebido: 22/5/2009

Revisado: $25 / 11 / 2009$

Aprovado: 28/12/2009

Trabalho realizado no Hospital Estadual Mário Covas de Santo André (SP), Brasil

Disciplina de Ortopedia e Traumatologia da Faculdade de Medicina do ABC, Santo André (SP), Brasil

Endereço para correspondência: Luciano Miller Reis Rodrigues - Rua Borges Lagoa, 1065, cj 40 - São Paulo (SP) - CEP 04038002 -

E-mail: luciano.miller@uol.com.br 


\section{Introdução}

A escoliose idiopática é o desvio mais comum encontrado na coluna vertebral, de etiologia desconhecida, sendo definida como um desvio da coluna no plano frontal ${ }^{1}$. O diagnóstico e o tratamento têm ocupado atenção especial no desenvolvimento da cirurgia ortopédica enquanto especialidade. Em suas formas mais brandas, a escoliose pode causar apenas mudança na forma do tronco, mas, quando grave, pode evoluir com comprometimento cardíaco e pulmonar.

A classificação da Sociedade de Pesquisa em Escoliose (SRS) recomenda que a escoliose idiopática seja classificada quando da idade do aparecimento da deformidade, devido à diferente evolução dos subtipos $^{2}$. A escoliose infantil ocorre entre o nascimento aos três anos de idade; a escoliose idiopática juvenil entre os quatro e dez anos; e a escoliose idiopática do adolescente, enfoque deste estudo, entre dez anos de idade e a maturidade esquelética.

As características da escoliose idiopática do adolescente incluem uma deformidade tridimensional da coluna vertebral com curvatura lateral mais a rotação dos corpos vertebrais ${ }^{3}$. A maioria das curvas idiopáticas são lordóticas ou hipocifóticas na região torácica, o que pode representar um fator importante em sua etiologia ${ }^{4}$.

Diversas técnicas cirúrgicas têm sido realizadas para a correção da deformidade ao longo dos anos, sendo que, mais recentemente, a correção com o uso de parafusos pediculares tem-se tornado uma referência para esse tratamento. Até a década de 1950, o princípio do tratamento baseava-se na realização de artrodese posterior, repouso no leito e imobilização. Harrington foi o primeiro a associar a instrumentação da coluna ${ }^{5,6}$. Em 1977, foi introduzido o sistema de Luque $^{7}$ com a utilização de fios sub-laminares, bilateralmente em cada segmento vertebral e sequencialmente apertados às hastes. A instrumentação posterior de terceira geração foi desenvolvida por Cotrel-Dubousset ${ }^{8}$ na década de 1980, utilizando-se hastes associadas a ganchos e parafusos pediculares; seus benefícios estão relacionados principalmente à maior correção nos planos frontal, sagital e na rotação vertebral ${ }^{9,10}$.

Assim, o objetivo deste estudo foi verificar o grau de correção obtido durante o tratamento cirúrgico da escoliose idiopática do adolescente, por meio da estabilização com parafusos pediculares.

Tabela I - Características gerais do grupo em estudo

\begin{tabular}{lll}
\hline & & Curva principal \\
\hline Número de pacientes & 21 pacientes & \\
Sexo masculino & 5 pacientes $(23,8 \%)$ & \\
Sexo feminino & 16 pacientes $(76,2 \%)$ & \\
Média de idade na cirurgia & $\begin{array}{l}15 \text { anos e } 2 \text { meses (vari- } \\
\text { ando de } 13 \text { a } 20 \text { anos) }\end{array}$ & 19 pacientes $(90,47 \%)$ \\
Número de curvas torácicas & 21 curvas & 2 pacientes $(9,53 \%)$ \\
\hline Número de curvas lombares & 21 curvas &
\end{tabular}

Método

Foram selecionados 21 pacientes com escoliose idiopática do adolescente, com indicação de tratamento cirúrgico, no período de 2006 a 2008 e com seguimento médio de 2 anos. Foram avaliados 16 mulheres e cinco homens com idade entre 13 e 20 anos. Consideraram-se critérios de inclusão a idade, na época do diagnóstico, (10 anos até a maturidade esquelética), a presença de escoliose idiopática, a indicação cirúrgica de correção por via posterior e a presença de curva lombar e/ou torácica. Os critérios de exclusão foram: presença de outra desordem neuromuscular, escoliose não-idiopática, necessidade de associação com via de acesso anterior, estudo radiológico incompleto. Esta pesquisa foi aprovada pelo comitê de ética da FMABC parecer 280/2007.

Todos foram submetidos à avaliação radiográfica (raio x de coluna nos planos frontal, sagital e frontal com flexão lateral direita e esquerda) e mensuração da deformidade pelo método de Cobb ${ }^{11}$. Esses pacientes também foram classificados conforme a rotação pedicular da vértebra ápice da curva por meio da classificação de Nash e Moe ${ }^{12} \mathrm{e}$ conforme o padrão da curva por meio da classificação de Lenke ${ }^{13}$.

Os pacientes realizaram acompanhamento ambulatorial semestral para avaliar a progressão da deformidade e só tiveram indicação cirúrgica quando o ângulo de Cobb ultrapassou $50^{\circ}$ e quando o tronco descompensado esteve presente.

Esses pacientes foram, então, submetidos a tratamento cirúrgico por via de acesso posterior com dissecção e exposição dos processos espinhosos e facetas bilateralmente articulares. Após isso, iniciou-se a colocação de parafusos pediculares através de parâmetros anatômicos e controle com radioscopia; a redução da deformidade foi realizada por meio do método de Cotrel-Dubousset ${ }^{8}$. A fixação estendeu-se desde a vértebra estável superior até a vértebra estável inferior da curva. Foram realizadas decorticação posterior e facetectomia, formando um leito para a artrodese o qual foi preenchido por enxerto autólogo do osso ilíaco.

No pós-operatório, esses pacientes realizaram novo estudo radiográfico (raio x de coluna nos planos frontal e sagital) com nova mensuração da deformidade por meio do método de $\mathrm{Cobb}^{11}$ e classificação da deformidade pelo método do Nash e Moe ${ }^{12}$. Foram feitas mensurações no pós-operatório imediato, após um mês, três meses, seis meses e anualmente de seguimento ambulatorial.

Os valores obtidos serão mostrados em forma de valor absoluto e relativo.

\section{Resultados}

Dos 21 pacientes selecionados, 16 eram mulheres (76,2\%) e 5 homens (23,8\%). A idade, no momento da cirurgia, variava de 13 a 20 anos. Os 21 pacientes apresentaram curvas torácicas e lombares, mas em apenas 2 casos (9,53\%) a curva lombar era a curva principal (Tabela I).

Em nosso estudo, a média do ângulo de Cobb pré-operatório para as curvas torácicas foi de $62,38^{\circ}$ (Figura 1), enquanto a média do 


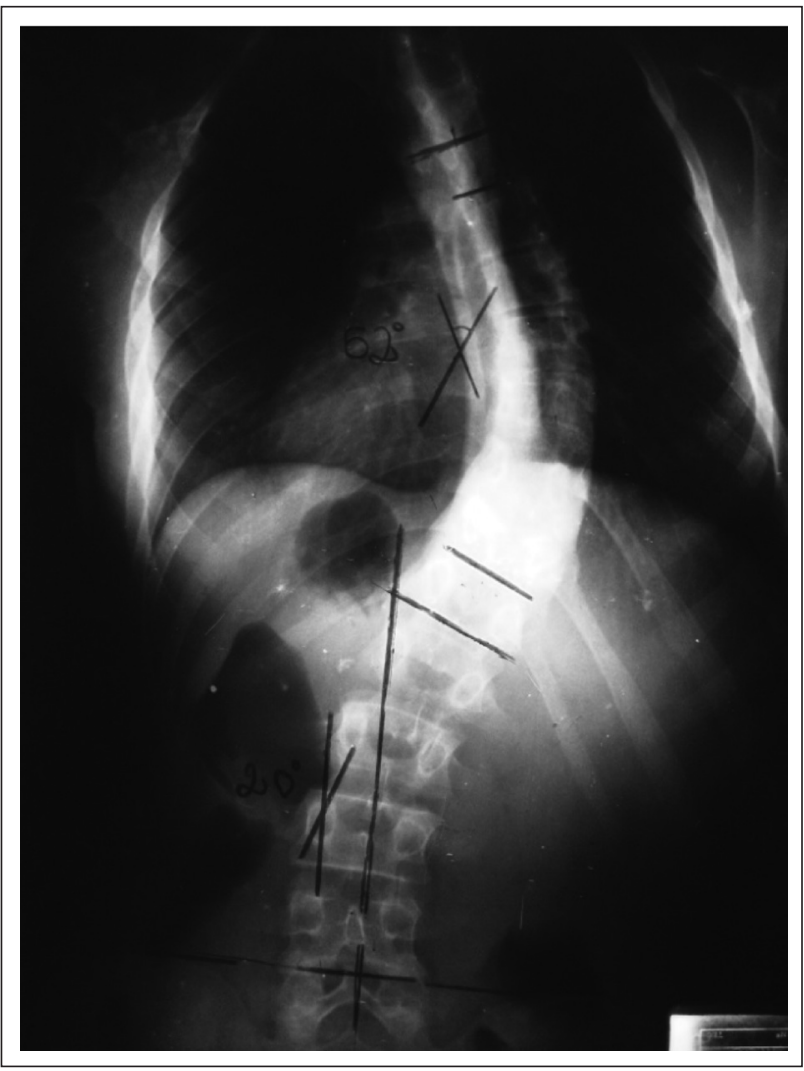

Figura 1 - Radiografia no plano frontal pré-operatória com ângulo de Cobb na curva torácica principal de $52^{\circ}$.

ângulo de Cobb pós-operatório foi de 38,28 (Figura 2). Já a média dos ângulos de Cobb pré-operatória para as curvas lombares foi de $40,52^{\circ}$, enquanto a média dos ângulos de Cobb pós-operatória foi de $21,74^{\circ}$ para as mesmas curvas lombares.

Esses resultados mostraram uma melhora média de $24,1^{\circ}$ nas deformidades pós-operatórias torácicas e de $18,78^{\circ}$ nas deformidades lombares. Isso reflete em uma melhora do ângulo de Cobb de 61,36\% para as curvas torácicas e de $53,65 \%$ para as curvas lombares, conforme apresentado na Tabela II.

Também foi mensurada, neste estudo, a variação da rotação vertebral pré e pós-operatória por meio da Classificação de Nash e Moe ${ }^{12}$. Obtivemos como resultado pré-operatório: 1) dois pacientes com classificação grau I; 2) 11 pacientes com grau II; 3) sete pacientes com grau III; e 4) um paciente com classificação grau IV.

Avaliando a classificação pós-operatória de Nash e Moe ${ }^{12}$ tivemos: 1) oito pacientes com classificação grau I; 2) 11 pacientes com grau II; 3) dois pacientes com grau III; e 4) nenhum paciente com grau IV (Tabela III).

Esses resultados refletem uma melhora de um grau na Classificação de Nash e Moe ${ }^{12}$ presente em 11 (52,38\%) pacientes do estudo. Nos demais 10 pacientes (47,62\%), não houve alteração do grau de rotação pré e pós-operatório de acordo com a Classificação de Nash e Moe ${ }^{12}$. Em nenhum caso houve piora da rotação após o tratamento cirúrgico com material de terceira geração (Tabela IV).

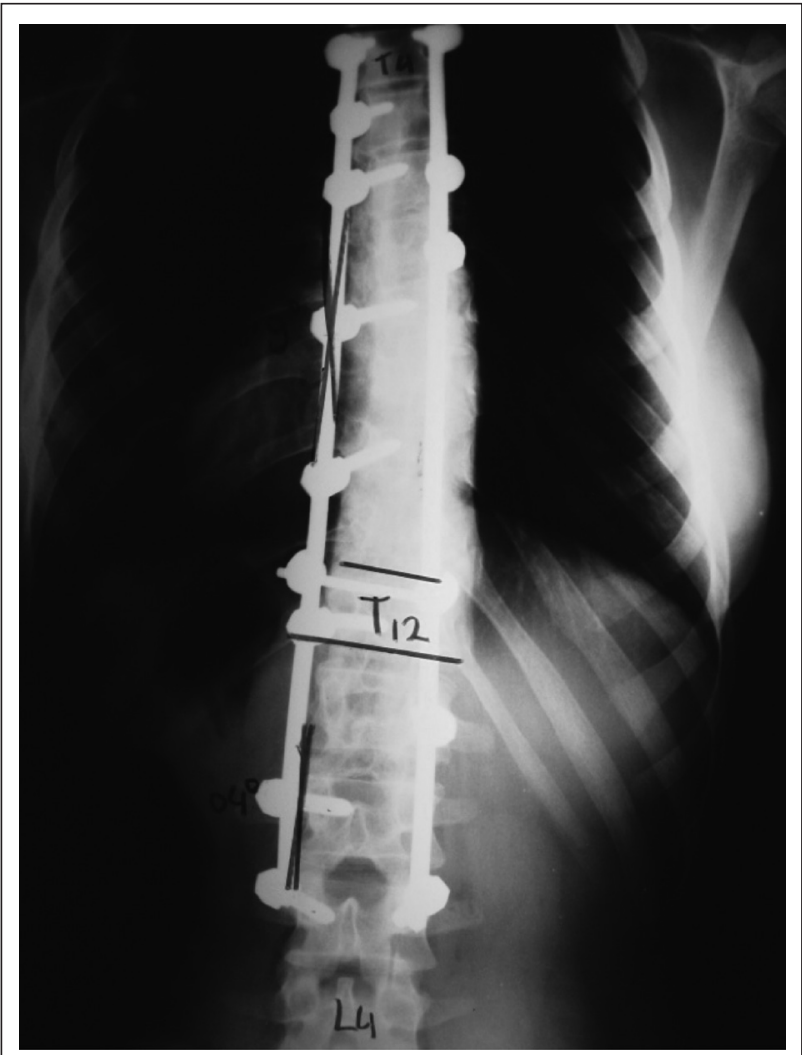

Figura 2 - Radiografia no plano frontal pós-operatória com ângulo de Cobb na curva torácica principal de $9^{\circ}$.

Tabela II - Medida dos valores pré e pós-operatorios

\begin{tabular}{lcc}
\hline & Curva torácica & Curva lombar \\
\hline $\begin{array}{l}\text { Média do ângulo de Cobb } \\
\text { Pré-operatória }\end{array}$ & $62,38^{\circ}$ & $40,52^{\circ}$ \\
$\begin{array}{l}\text { Media do ângulo de Cobb } \\
\text { Pós-operatória }\end{array}$ & $38,8^{\circ}$ & $21,74^{\circ}$ \\
$\begin{array}{l}\text { Melhora do ângulo de Cobb } \\
\text { em graus }\end{array}$ & $24,1^{\circ}$ & $18,78^{\circ}$ \\
$\begin{array}{l}\text { Melhora do ângulo de Cobb } \\
\text { em porcentagem }\end{array}$ & $61,36 \%$ & $53,66 \%$ \\
\hline
\end{tabular}

Tabela III - Resultados da classificação de Nash e Moe ${ }^{12}$ pré e pós-operatórios

\begin{tabular}{lcccc} 
& Grau I & Grau II & Grau III & Grau IV \\
\hline $\begin{array}{l}\text { Classificação } \\
\text { de Nash e Moe } \\
\text { pré-operatório }\end{array}$ & 2 pacientes & 11 pacientes & 7 pacientes & 1 paciente \\
$\begin{array}{l}\text { Classificação de } \\
\text { Nash e Moe pós- } \\
\text { operatório }\end{array}$ & 8 pacientes & 11 pacientes & 2 pacientes & 0 paciente
\end{tabular}

Tabela IV - Resultados da melhora da classificação de Nash e Moe ${ }^{12}$ pós-operatórios

\begin{tabular}{lcc}
\hline & $\mathrm{N}^{0}$ de pacientes & Porcentagem \\
\hline Com melhora da rotação vertebral & 11 pacientes & $52,38 \%$ \\
$\begin{array}{l}\text { Mantendo rotação vertebral pré- } \\
\text { operatória }\end{array}$ & 10 pacientes & $47,62 \%$ \\
Com piora da rotação vertebral & 0 paciente & $0 \%$ \\
\hline
\end{tabular}




\section{Discussão}

O manejo cirúrgico da escoliose tem passado por uma evolução muito dinâmica nos últimos 50 anos, com uma rápida explosão de novas técnicas nos últimos 15 anos. Desde os primórdios de 1900 até o final da década de 1950, a fusão posterior sem instrumental foi o tratamento padrão. A instrumentação vertebral introduzida por Paul Harrigton ${ }^{6}$, associada à artrodese vertebral, foi o maior passo no tratamento cirúrgico da escoliose. O instrumental de Harrington ${ }^{6}$ usava forças de distração e compressão para correção das deformidades da coluna vertebral. Esse sistema trouxe avanços, pois promovia boa correção da deformidade, mas frequentemente estava associada ao uso de órtese ou gesso pósoperatório.

No final da década de 1970, Luque e Cardoso 7 introduziram os fios sublaminares à instrumentação, dando início, com isto, à primeira instrumentação segmentar, introduzindo o conceito de translação e desrotação, em vez de distração como principal mecanismo de correção da curva.

Durante a década de 1980, o conceito de instrumentação segmentar foi expandido para incluir múltiplos ganchos e hastes ao instrumental. Durante a década de 1990, Kim et al. ${ }^{9}$ demonstraram que, com o uso de parafusos pediculares, foi possível maior correção da curva e melhora da função pulmonar comparando-se com a instrumentação híbrida (ganchos e parafusos).

Embora a maioria dos autores recomende a cirurgia quando a curva atinge $50^{\circ}$, outros fatores necessitam ser considerados, como desvio do tronco, descompensação coronal e deformidade cosmética. Curvas duplas de $50^{\circ}$ não são tão inaceitáveis cosmeticamente quanto as curvas simples; e, se ocorrer progressão em pacientes esqueleticamente maduros, é provável que seja gradual ${ }^{14}$. Neste trabalho consideramos $50^{\circ}$ como o valor mínimo para indicação de tratamento cirúrgico.

Kadoury et al ${ }^{15}$ avaliaram o grau de correção da escoliose nos três planos da deformidade, comparando-o à instrumentação de HarringtonLuque $^{6,7}$ e de Cotrel-Dubousset ${ }^{8}$ (instrumentação de terceira geração com parafusos pediculares e derotação vertebral direta). Os resultados indicaram que a correção espinhal no plano coronal seguiu a mesma ten- dência nos três tipos de instrumentação; no entanto, tiveram uma correção significantemente maior na região lombar da coluna usando a técnica de Cotrel-Dubousset ${ }^{8}$ comparada com a haste de Harrington-Luque ${ }^{6,7}$. Já a correção espinhal no plano sagital com instrumental de Cotrel-Dubousset $^{8}$ e parafusos pediculares de terceira geração muitas vezes restabelece os valores fisiológicos de cifose torácica e lordose lombar.

Em relação aos resultados apresentados neste trabalho, encontramos forte relação de semelhança com a literatura vigente. Obtivemos $16(76,2 \%)$ pacientes do sexo feminino e $5(23,8 \%)$ pacientes do sexo masculino com diagnóstico de escoliose idiopática, o que confere com os resultados de Kadoury et al..$^{15}$ que tiveram $83,9 \%$ dos resultados em mulheres contra $16,1 \%$ em homens, confirmando a forte prevalência de escoliose idiopática do adolescente no sexo feminino. Em relação à idade do paciente no momento da cirurgia, tivemos uma média de idade de 15 anos e 2 meses, valor muito próximo do também obtido por Kadoury et al. ${ }^{15}$, que foi de 15,3 anos com variação de $\pm 2,3$ anos.

Analisando a melhora média do ângulo de Cobb dos pacientes submetidos a tratamento cirúrgico tivemos uma melhora de $24,1^{\circ}$ $(61,36 \%)$ para as curvas torácicas e melhora de $18,78^{\circ}(53,66 \%)$ para as curvas lombares, resultados estes, próximos dos obtidos por Hempfing et al. ${ }^{10}$, com média de correção de $25,2^{\circ}(71,9 \%)$.

Sobre a variação da classificação de Nash e $\mathrm{Moe}^{12}$ no pré e pósoperatório, tivemos melhora mensurável da rotação vertebral em 11 $(52,38 \%)$ pacientes, comprovando que a redução com método de Cotrel-Dubousset ${ }^{8}$ e estabilização com parafusos pediculares de terceira e quarta gerações melhoram a deformidade escoliótica, tanto no plano sagital como no coronal e rotacional.

Alguns autores advertem o uso de parafusos pediculares na curvatura torácica em relação à segurança, sendo que, em trabalho recente, Maruyama et al. ${ }^{16}$ demonstraram uma grande segurança na técnica de colocação de parafusos pediculares torácicos e sua relação anatômica com os grandes vasos e o canal intervertebral.

Concluímos, ainda, que a fixação com parafusos pediculares, tanto na região lombar quanto torácica, mostrou-se um método seguro com bom grau de correção nos planos sagital, coronal e rotacional.

\section{Referências}

1. Lonstein JE. Idiopathic scoliosis. In: Lonstein JE. Moe's textbook of scoliosis and other deformities. 3a ed. Philadelphia: Saunders; 1994. p. 219-56.

2. Barney L, Freeman III. Kyphosis and scoliosis. In: Canale ST. Campbell's operative orthopaedics . 10a ed. New York: Elsevier Health Sciences; 2003. p. 1755.

3. King HA. Idiopatic scoliosis. In: Herkowitz HN, Gafin SR, Esimont FJ, Bell GR, Balderston RA, Rothman S. The spine. 5a ed. Philadelphia: Saunders; 2006.

4. Dickson RA, Deacon P. Spinal growth. J Bone Joint Surg Br. 1987;69(5): 690-2.
5. Green NE.The role of Harrington rods and Wiscosin wires in idiopathic scoliosis. In: Bridwell KH, DeWald RL, editors. The textbook of spinal surgery. 2nd ed. St.Louis: Lippincott; 1996. p. 249-65.

6. Harrington PR. Treatment of scoliosis. Correction and internal fixation by spine instrumentation. J Bone Joint Surg Am. 1962;44:591-610.

7. Luque ER, Cardoso A. Segmental correction of scoliosis with rigid internal fixation. Orthop Trans. 1977:1:136-7.

8. 8. Cotrel Y, Dubousset J. Nouvelle technique d'osthéosynthèse rachidienne segmentaire par voie postérieure. Rev Chir Orthop Reparatrice Appar Mot. 1984;6:489 
9. Kim YJ, Lenke LG, Cho SK, Bridwell KH, Sides B, Blanke K. Comparative analysis of pedicle screw versus hook instrumentation in posterior spinal fusion of adolescent idiopathic scoliosis. Spine (Phila Pa 1976). 2004;29(18):2040-8.

10. Hempfing A, Ferraris L, Koller H, Rump J, Metz-Stavenhagen P. Is anterior release effective to increase flexibility in idiopathic thoracic scoliosis? Assessment by traction films. Eur Spine J. 2007;16(4): 515-20.

11. Cobb JR. Outline for the study of scoliosis. In: Edwards JW, editor. Instructional Course Lectures. Ann Arbor: The American Academy of Orthopaedic Surgeons. 1948;5:261-75.

12. Nash CL Jr, Moe JH. A study of vertebral rotation. J Bone Joint Surg Am. 1969;51(2):223-9.
13. Lenke LG, Betz RR, Harms J, Bridwell KH, Clements DH, Lowe TG et al. Adolescent idiopathic scoliosis: a new classification to determine extent of spinal arthrodesis. J Bone Joint Surg Am. 2001;83A(8):1169-81.

14. Dickson JH. An eleven-year clinical investigation of Harrington instrumentation. A preliminary report of 578 cases. Clin Orthop Relat Res. 1973;(93):113-30.

15. Kadoury $S$, Cheriet $F$, Beauséjour $M$, Stokes IA, Parent $S$, Labelle $H$. A three-dimensional retrospective analysis of the evolution of spinal instrumentation for the correction of adolescent idiopathic scoliosis. Eur Spine J. 2009;18(1):23-37.

16. Maruyama T, Takeshita K, Nakamura K, Kitagawa T. Spatial relations between the vertebral body and the thoracic aorta in adolescent idiopathic scoliosis. Spine (Phila Pa 1976). 2004;29(18):2067-9. 